\title{
Pedagogía de la muerte: estudio sobre la ansiedad ante la muerte en profesionales de la educación
}

\section{Pedagogy of death: a study on death anxiety in education professionals}

\author{
Ernesto Colomo Magaña ${ }^{1}$, Vicente Gabarda Méndez ${ }^{2}$, Patricia Motos Sellés ${ }^{3}$ \\ ${ }^{1}$ Universidad Internacional de Valencia, España (ecolomo@universidadviu.com) \\ ${ }^{2}$ Universidad Internacional de Valencia, España (vgabarda@universidadviu.com) \\ ${ }^{3}$ Universidad Internacional de Valencia, España (pmotos@universidadviu.com)
}

\section{RESUMEN:}

Pese a que la muerte forma parte de la vida y es una realidad inevitable por las que todos pasamos en algún momento, aún no se ha conseguido naturalizar $\mathrm{y}$ sigue siendo un hecho que provoca ansiedad y miedo. La muerte es una realidad que los docentes de hoy en día deben poder abordar en las aulas. En este sentido, este trabajo analiza el grado de ansiedad ante la muerte de 64 universitarios del Grado en Educación Primaria, mediante el cuestionario DAI (Death Anxiety Inventory).

Los resultados muestran que la mayoría de los entrevistados manifiestan una ansiedad considerable ante esta circunstancia, coincidiendo con aportaciones de estudios anteriores con otras poblaciones, incidiendo en la necesidad de incluir una formación universitaria centrada en la pedagogía de la muerte, con el fin de reducir dichos niveles de ansiedad y aportar herramientas para conseguir docentes más competentes en los procesos de duelo dentro del ámbito escolar.

PALABRAS CLAVE: ANSIEDAD, MUERTE, FORMACIÓN DOCENTE

\begin{abstract}
:
Although death is a part of live and is an unavoidable reality that we all encounter at some point, it has not yet been able to naturalize and it is still a fact that provokes anxiety and fear. Currently, death is a reality that teachers must be able to address in the classroom. In this sense, this paper analyses the degree of anxiety towards death for 64 undergraduate students carrying out a degree of Primary Education. The study makes use of the DAI questionnaire (Death Anxiety Inventory).

The results show that most of the interviewees show considerable anxiety regarding this situation. These results coincide with contributions from previous studies with other populations that consider including a university education focused on the pedagogy of death, in order to reduce the levels of anxiety and provide tools that will make our teachers more competent in grieving processes in school settings.
\end{abstract}

KEYWORDS: ANXIETY, DEATH, TEACHER TRAINING 


\section{INTRODUCCIÓN}

El miedo a la muerte es un miedo universal (KüblerRoss, 2006, p.18).

A pesar de que la muerte es una realidad presente en nuestro día a día, los maestros afirman no estar preparados adecuadamente para acompañar a los educandos en sus procesos de duelo (Adamson y Peacock, 2007; Brown, Jimerson, Comerchero, 2015), por lo que cualquier pérdida dentro de la comunidad escolar se convierte en un evento traumático (Pereira-Webber y Pereira-Webber, 2014), interfiriendo directamente en la vida del centro educativo. La finitud de la existencia humana, a nivel educativo, sigue estando apartada y convertida en un tema tabú que se tiende a obviar (Puolimatka y Solasaari, 2006), lo que conlleva su invisibilidad en el contexto familiar, escolar e, incluso, en la educación superior (Gaona, 2015).

La conciencia de la condición mortal de todo ser humano influye en la construcción identitaria, generando con frecuencia una respuesta emocional adversa ante dicha realidad. En este sentido, una de las variables más estudiadas dentro de este ámbito es la ansiedad ante la muerte (Limonero, TomásSábado, Fernández-Castro, Cladellas y GómezBenito, 2010). El motivo principal de la aparición de este estado emocional es la presencia del factor miedo a nivel social, ya que, pese a que la muerte es una realidad inherente en nuestra existencia, genera ansiedad el desconocimiento en torno a la misma (Moreno, 2013) y el que sea una realidad no controlable ni evitable (Ramos, Gairín y Camats, 2018).

Estos niveles de ansiedad ante la muerte, aunque están constatados en diferentes poblaciones, sobre todo entre el personal sanitario (Cavaye y Watts, 2014; Peters et al., 2013), no lo están entre el colectivo docente. Este último grupo, pese a tener un contacto directo y continuo con menores de edad, y que por necesidad la muerte va a estar presente en su lugar de trabajo, carecen de investigación al respecto. Ello, unido a la falta de formación inicial que sobre esta temática tienen los futuros profesionales de la educación, conlleva la aparición y mantenimiento de estados de incertidumbre ante las situaciones de duelo que puedan acaecer en el centro escolar y en los modos de actuar por parte de los diferentes agentes educativos. Por todo ello, surge la necesidad de conocer el grado de ansiedad de los futuros docentes (egresados de magisterio), para incluir en la formación universitaria herramientas y destrezas que permitan la reducción de los niveles de ansiedad ante la muerte. Ello, permitiría conseguir docentes más competentes para intervenir, de manera eficaz, tanto de manera preventiva como paliativa dentro del ámbito escolar.

Para poder analizar el nivel de ansiedad ante la muerte es necesario la implementación de instrumentos que evalúen dicha variable. En este estudio se ha utilizado el Death Anxiety Inventory (DAI), por ser una de las herramientas más utilizadas en nuestro país, tanto en el colectivo de enfermería (Aradilla y Tomás-Sábado, 2006; Tomás-Sábado y Limonero, 2004) como en otros grupos poblacionales: estudiantes de secundaria, universitarios, operarios, profesores, doctores $\mathrm{o}$ pensionistas (Tomás-Sábado y Gómez-Benito, 2005), obteniendo en todos los casos adecuadas propiedades psicométricas.

Por todo ello, en el presente trabajo se pretende realizar una exploración inicial sobre los niveles de ansiedad ante la muerte en una población de estudiantes universitarios del Grado en Educación Primaria, con el fin de aportar mayor información sobre el estado de ansiedad que se encuentra este colectivo en situaciones donde la muerte está presente.

\section{FUNDAMENTACIÓN TEÓRICA}

\subsection{Ansiedad ante la muerte}

La muerte es un hecho inevitable, personal e intransferible, cuya certeza reside en cada sujeto. Pese a dicha evidencia, existe un temor inherente en la condición humana a la finitud de la vida. Cabadas (2004) habla del miedo a la muerte como un miedo ancestral ante lo desconocido que los seres humanos no han conseguido superar. Como recogen Ramos, et al. (2018), "la muerte provoca sufrimiento y, por tanto, origina emociones que no deseamos [...] morir se convierte en algo terrorífico porque supone la pérdida de todo" (p. 22). Esta realidad se hace patente en diferentes estudios. En el trabajo de López (2016), con una muestra de 825 estudiantes (51.2\% universitarios y $48.8 \%$ pertenecientes a educación secundaria), se observó que el 23\% manifestaba una actitud de miedo a la muerte. Estos datos son más significativos en un estudio anterior realizado por Ransanz (2014) en el que el porcentaje de estudiantes universitarios que reconoció tener miedo a la muerte superó el $82 \%$.

Los resultados reflejan que el temor a la muerte es una reacción emocional habitual y normal del ser humano, fruto del instinto de supervivencia. Sentir cierta ansiedad ante aquello que envuelve a la mortalidad es algo natural, como ocurre con el miedo a morir, el sufrimiento que provoca una 
enfermedad, lo que ocurre después de la muerte, etc. (Aradilla y Tomás-Sábado, 2006). Dicha reacción emocional es producida por la percepción de señales de peligro o amenaza, ciertas o imaginadas, a la propia existencia (Limonero, 1997), definiéndose en la literatura como ansiedad ante la muerte. Este constructo se utiliza para conceptualizar la aprehensión generada por la conciencia de la muerte (Abdel-Khalek, 2005), produciéndose al tomar mayor conciencia de la relevancia que la muerte tiene para los individuos como seres finitos (Letho y Farchaus, 2009). Puede desencadenarse ante estímulos ambientales, como cadáveres, cementerios, etc.; estímulos situacionales, los cuales se hayan condicionado por asociación con los anteriores y son capaces de provocar una respuesta condicionada; y estímulos internos de la persona, como pensamientos o evocación de imágenes relacionadas con la muerte propia o ajena (TomásSábado y Gómez-Benito, 2003).

Se trata de un factor presente en la población en diferentes niveles a tenor del grado de asimilación de la condición mortal. Pese a ello, la sociedad de hoy en día se caracteriza por una polarización del sistema de valores en el que el envejecimiento, la enfermedad y la muerte se consideran un fracaso, por lo que este tipo de temas no son abordados socialmente (Tomás-Sábado y Gómez-Benito, 2003). Esto provoca que las personas no desarrollen, de una manera normalizada, estrategias de afrontamiento ante este tipo de situaciones que inevitablemente experimentarán a lo largo de su vida, acarreando mayores niveles de ansiedad.

\subsection{Presencia de la muerte en la pedagogía}

Partiendo de que el fenómeno de la muerte es importante para todas las personas, se considera especialmente relevante para el personal docente, debido a la presencia del factor humano en las diferentes acciones que realizan y por su trabajo para con un grupo poblacional cuya etapa evolutiva les dificulta la comprensión de algunos temas relacionados con el sentido de la vida y la presencia de la muerte en la misma.

En nuestro país, el abordar la muerte en el campo educativo sigue siendo una temática emergente, sin tradición profesional en didáctica y pedagogía (Rodríguez, Herrán y Cortina, 2015). Esta situación subraya la necesidad de su inclusión en dicho campo, como atestiguan varios estudios. Colomo y Cívico (2018), indican que el 88\% de estudiantes del máster en formación del profesorado nunca había recibido una formación específica sobre el tratamiento pedagógico de la muerte y el duelo. Por su parte, Pedrero (2012) realizó un estudio con 455 estudiantes universitarios de las titulaciones de Trabajo Social, Educación Social y la Titulación Conjunta de Trabajo Social y Educación Social. Entre los resultados, cabe destacar que más del $80 \%$ no había recibido información/formación sobre educación para la muerte, del cual cerca del $60 \%$ manifestó tener necesidad de recibir formación en este ámbito. Datos semejantes nos muestra el trabajo de Ransanz (2014), en el que más de la mitad $(53.33 \%)$ de los estudiantes del área de educación, estaba de acuerdo en que formara parte de la formación inicial, ascendiendo dicho acuerdo al $60 \%$ cuando se aludía a la formación continua del profesorado. Destacar la unanimidad (100\%), recogida en el estudio de Colomo y Cívico (2018), en el que la muestra de 250 universitarios coincidió en remarcar la necesidad de incluir esta formación para el desarrollo de la labor docente.

No obstante, van surgiendo progresivamente estudios y propuestas centradas en cuatro núcleos principales (Rodríguez, Herrán y Cortina, 2012): en el valor formativo de la muerte para la evolución como ámbito perenne del ser humano, en la normalización de la muerte en la educación, en la intervención educativa paliativa o en el análisis de experiencias didácticas y de formación de profesorado. Especialistas como Poch (2009), argumentan que abordar el tema de la muerte en la escuela no puede ser fruto de la improvisación. Por ello, son múltiples las propuestas, recursos o materiales diseñados para este fin y varios los autores (Colomo y Oña, 2014; Colomo, 2016; Cortina y Herrán, 2011; Feijoo y Pardo, 2003; Herrán y Cortina, 2009; Poch y Herrero, 2003) que destacan el papel del educador por su vínculo directo con el discente, tanto en el acompañamiento en las situaciones de duelo (trabajo paliativo) como en el proceso formativo para concienciar sobre la finitud humana (trabajo preventivo). La muerte forma parte de la vida y es una realidad que no puede quedar al margen del contexto pedagógico (Wass, 2004). Existe la esperanza, y se está trabajando, para que este ámbito acabe ocupando un hueco vital en el currículo como tema esencial para el ser humano (Pedrero y Muñoz, 2008). La mayoría de estos contenidos se trabajan con fines preventivos, para que la formación se anticipe a las situaciones de duelo en el futuro, dando respuesta a las dificultades que estas conllevan y estando más preparados para afrontarlas. Este proceso, como recoge Ramos (2016), da la oportunidad al educando de aprender sobre la muerte sin tener que gestionar a la vez todas las emociones que ya de por sí comporta el duelo. El fin último de la Pedagogía de la Muerte es que las 
personas se centren en vivir una existencia plena en todos los aspectos y sentidos, siendo conscientes de que su finitud es un hecho inevitable (Enseñar a vivir completamente; Aprender a superar los temores; Normalizar la muerte, siendo conscientes de su finitud). Si queremos un desarrollo integral y holístico de nuestros educandos respecto a su construcción identitaria, "necesitamos tanto educación para la muerte como para la vida" (Osho, 2004, p.45).

Sin embargo, una implementación de calidad sobre esta materia, y de los instrumentos y contenidos que se van diseñando para el ámbito escolar, conlleva obligatoriamente una formación específica y de calidad para los profesionales de la educación. Se trata de una apuesta decidida por formar a los docentes para trabajar la muerte dentro del contexto educativo. Para ello, dentro del currículo universitario de las diferentes titulaciones educativas, sería necesario generar un espacio para la formación en el ámbito de la muerte. El fin es dotar a estos futuros profesionales de los conocimientos, competencias, habilidades, destrezas y herramientas para trabajar sobre el duelo y la finitud de la vida, tanto de manera preventiva como paliativa, en los diferentes contextos de enseñanzaaprendizaje.

Por todo ello, en el presente trabajo se estudiará el papel de la ansiedad ante la muerte en una muestra de estudiantes universitarios del grado de primaria. Averiguar los niveles de ansiedad ante la muerte de futuros docentes ayudará a proponer estrategias de intervención más ajustadas a las necesidades de dicha población, permitiendo una reducción de la misma.

\section{MATERIAL Y MÉTODO}

\subsection{Participantes}

La muestra objeto de estudio está conformada por estudiantes del Grado de Educación Primaria de la Universidad Internacional de Valencia (VIU) que cursan la asignatura Derechos Humanos y Educación en un mundo globalizado, durante el curso académico 2016/2017. La totalidad de la población consta de 91 estudiantes $(\mathrm{N}=91)$. El número de estudiantes que participaron en el estudio y contestaron el cuestionario es de $64(70,3 \%)$. En dicha muestra, un $89,1 \%$ son mujeres (57) y un $10,9 \%$ son hombres (7).

La edad está comprendida entre los 20 y 49 años $(\mathrm{Md}=33.2$ años y $\mathrm{DE}=6.92)$. Un rango de edad acorde al perfil de estudiantes que cursan estudios universitarios a través de la metodología e-learning.
En el presente estudio se utilizó un diseño trasversal de tipo descriptivo. La muestra fue seleccionada a través de un muestreo no probabilístico, del tipo de conveniencia o causal (Alaminos, 2006; Sabariego, 2012) que se basa en la facilidad de acceso por parte del investigador a los participantes del estudio. En este caso los investigadores eran profesores de la materia Derechos Humanos y Educación en un mundo globalizado de VIU.

\subsection{Procedimiento}

El criterio para la selección de los sujetos de la muestra de estudio fue la cumplimentación de un cuestionario autoadministrado de manera voluntaria y anónima, a través de la aplicación Google Forms (on-line), por su flexibilidad y capacidad para ajustarse a las necesidades de la investigación de forma gratuita y sin limitaciones (Abundis, 2016).

Previamente, se llevó a cabo una sesión informativa para explicar al alumnado que el objetivo del estudio era analizar los niveles de ansiedad que pueden generar ciertas situaciones relacionadas con la muerte. En todo momento, se les aseguró el anonimato y la confidencialidad de los datos. Todos aquellos interesados en participar dieron su consentimiento informado.

\subsection{Instrumento}

Para evaluar la ansiedad ante la muerte, se aplicó una adaptación del cuestionario DAI (Death Anxiety Inventory) realizada por Tomás-Sábado y GómezBenito (2005), la cual posee un coeficiente $\alpha$ de consistencia interna de 0,90 , convirtiéndolo en un instrumento psicométrico válido y fiable para la evaluación. Incluye 20 ítems agrupados en cinco factores significativos ${ }^{1}$ : Generadores externos de ansiedad ante la muerte (1); Significado y aceptación de la muerte (2); Pensamiento sobre la muerte (3); Vida después de la muerte (4); y Brevedad de la vida (5). Para su cumplimentación, se utiliza una escala tipo Likert con cinco opciones: total acuerdo, moderado acuerdo, ni acuerdo ni desacuerdo, moderado desacuerdo, total desacuerdo. La puntuación siempre es en positivo, oscilando entre 5 puntos para el total acuerdo y 1 punto para el total desacuerdo. El rango de resultado del total de la escala varía entre 20 puntos (el más bajo) y 100 puntos (el más alto), y el de los factores desde 4 puntos (el más bajo) hasta 20 puntos (el más alto). A mayor puntuación mayores niveles de ansiedad ante la muerte. Junto con el cuestionario DAI, los participantes respondieron también a algunos datos 
sociodemográficos personales, tales como el sexo y la edad.

\subsection{Análisis de datos}

Mediante el paquete estadístico IBM SPSS Statistics 20 se llevaron a cabo análisis descriptivos de las puntuaciones del cuestionario DAI, de sus cinco factores y de los ítems que los componen. También se calculó el alfa de Cronbach para analizar la consistencia interna de la escala en la muestra de estudio.

\section{RESULTADOS}

En primer lugar, se calculó el índice de alfa de Cronbach para comprobar la consistencia interna del conjunto de ítems de la escala DAI en esta población. El resultado muestra un valor de .903 (0.7 mínimo de fiabilidad aceptado), que se traduce en un coeficiente de alfa excelente, por lo que garantiza la fiabilidad de la escala en este grupo poblacional concreto.

A continuación, se presentan las puntuaciones medias de la escala total del DAI así como de sus factores (tabla 1). Los resultados indican un valor medio de 53.63 $(\mathrm{DE}=15.391)$ para la escala general, considerándose un nivel de ansiedad moderado de la población. Cuando se atiende a los cinco factores de la escala, las puntuaciones más altas se observan en los factores que hacen referencia en primer lugar a los agentes externos que pueden provocar ansiedad ante la muerte y en segundo lugar a la significación y aceptación ante la muerte. Ambos presentan puntuaciones moderadas altas. Les siguen con unos niveles moderados bajos los factores que miden el pensamiento ante la muerte y la vida después de la muerte. Por último, se encuentra el factor asociado a pensamientos sobre la brevedad de la vida con una puntuación baja.
Tabla 1. Puntuaciones medias de la escala total del DAI y de sus cinco factores

\begin{tabular}{|c|c|c|c|c|}
\hline & Media & $\begin{array}{l}\text { Desviación } \\
\text { Estándar }\end{array}$ & Mínimo & Máximo \\
\hline $\begin{array}{l}\text { Puntuación } \\
\text { total del } \\
\text { DAI }\end{array}$ & 53,63 & 15,391 & 27 & 89 \\
\hline $\begin{array}{l}\text { Generadores } \\
\text { externos de } \\
\text { ansiedad } \\
\text { ante la } \\
\text { muerte }\end{array}$ & 14,63 & 4,530 & 6 & 25 \\
\hline $\begin{array}{l}\text { Significado } \\
\text { y aceptación } \\
\text { ante la } \\
\text { muerte }\end{array}$ & 13,70 & 4,268 & 5 & 25 \\
\hline $\begin{array}{l}\text { Pensamiento } \\
\text { ante la } \\
\text { muerte }\end{array}$ & 10,27 & 4,048 & 4 & 20 \\
\hline $\begin{array}{l}\text { Vida } \\
\text { después de } \\
\text { la muerte }\end{array}$ & 10,27 & 4,048 & 4 & 20 \\
\hline $\begin{array}{l}\text { Brevedad de } \\
\text { la vida }\end{array}$ & 7,05 & 2,675 & 3 & 13 \\
\hline
\end{tabular}
pormenorizado, abordando los diferentes ítems que componen el DAI (Tabla 2), se contempla que la puntuación más alta se ha registrado en el ítem vinculado con el deseo de vivir durante mucho tiempo que pertenece al factor que hace referencia al significado y aceptación ante la muerte. Le siguen los ítems sobre el rechazo a trabajar en una empresa funeraria y sobre la visión de un cadáver, ambos pertenecientes al factor generadores externos de ansiedad ante la muerte. Por último, cabe destacar el ítem relacionado con la preocupación que provoca la posibilidad de morir jóvenes y el ítem asociado a la dificultad para aceptar la idea de que todo acabe con la muerte, pertenecientes al tercer factor que mide el pensamiento ante la muerte y el cuarto factor que evalúa el pensamiento sobre la posibilidad de que haya vida después de la muerte, respectivamente. En cuanto a las puntuaciones más bajas obtenidas en el DAI, la muestra ha coincidido en valorar por debajo del moderado desacuerdo el ítem acerca de que la muerte quita significado a la vida (primer lugar), y el ítem que evalúa la creencia de tener más miedo a la muerte que la mayoría de personas (segundo lugar). 
Tabla 2. Puntuaciones medias de los 20 ítems que componen la escala DAI

\begin{tabular}{lcccc}
\hline Ítems & Media & $\begin{array}{c}\text { Desviación } \\
\text { Estándar }\end{array}$ & Mínimo & Máximo \\
\hline I1 & 2,33 & 1,196 & 1 & 5 \\
I2 & 1,84 & 1,011 & 1 & 4 \\
I3 & 2,50 & 1,272 & 1 & 5 \\
I4 & 3,17 & 1,464 & 1 & 5 \\
I5 & 3,03 & 1,563 & 1 & 5 \\
I6 & 2,55 & 1,425 & 1 & 5 \\
I7 & 1,92 & 1,103 & 1 & 5 \\
I8 & 2,66 & 1,263 & 1 & 5 \\
I9 & 2,30 & 1,164 & 1 & 5 \\
I10 & 3,50 & 1,469 & 1 & 5 \\
I11 & 2,39 & 1,399 & 1 & 5 \\
I12 & 2,83 & 1,279 & 1 & 5 \\
I13 & 2,39 & 1,341 & 1 & 5 \\
I14 & 4,17 & 1,092 & 1 & 5 \\
I15 & 2,83 & 1,304 & 1 & 5 \\
I16 & 2,56 & 1,220 & 1 & 5 \\
I17 & 2,55 & 1,284 & 1 & 5 \\
I18 & 2,48 & 1,368 & 1 & 5 \\
I19 & 3,47 & 1,425 & 1 & 5 \\
I20 & 2,16 & 1,185 & 1 & 5 \\
\hline
\end{tabular}

\section{DISCUSIÓN Y CONCLUSIONES}

En primer lugar, se confirma la alta fiabilidad que presenta la escala en esta población, alcanzando incluso un valor alfa superior al de estudios anteriores con estudiantes de enfermería (TomásSábado y Gómez-Benito, 2003, 2005). De ello, se deduce que nos encontramos ante un instrumento de medida efectivo para evaluar la reacción emocional de ansiedad ante la muerte en esta población concreta.

Los resultados muestran la existencia de cierta tendencia a experimentar ansiedad ante todo aquello que acontece a la muerte entre los participantes del estudio. Principalmente, en aquellos factores de la escala que hacen referencia a la vulnerabilidad que presenta esta población para experimentar ansiedad derivada de la percepción de estímulos relacionados con la muerte y a la dificultad que tienen para comprender el significado de la muerte.

Concretamente, la muestra obtiene una puntuación total del grado de ansiedad ante la muerte igual o incluso superior a la de otros estudios previos con otro tipo de poblaciones. Concretamente, el trabajo de Aradilla-Herrero et al. (2006) muestra una puntuación media del DAI de 40,32 con personal de enfermería. Limonero, Tomás-Sábado y Fernández-Castro (2006) indican un valor algo superior con población universitaria del grado de psicología, alcanzando una media de 53 puntos. En ambos casos las puntuaciones son inferiores a la de la muestra de este estudio.

Por este motivo, al igual que se ha realizado en estudios previos con otras poblaciones, surge la necesidad de elaborar estrategias educativas sobre pedagogía de la muerte ajustadas a las necesidades de la población estudiantil de magisterio. Concretamente, en el colectivo evaluado en el presente trabajo sería conveniente aumentar el nivel de comprensión del proceso de la muerte y de su significado en la vida, porque solo así serán capaces de asumir sus propias emociones sobre la muerte, lo que ayudará a reducir paralelamente el grado de ansiedad generado por cuestiones tales como la incertidumbre por lo qué sucede después de la muerte o la angustia por la posibilidad de morir jóvenes. Asimismo, será necesario proporcionarles mecanismos de afrontamiento que reduzcan los niveles de ansiedad ante estímulos o situaciones externas relacionadas con sucesos terminales.

Por tanto, nos encontramos hoy en día en una realidad en la que los profesionales de la educación presentan unos niveles de ansiedad que imposibilitan afrontar de manera adecuada las situaciones de duelo en el ámbito escolar, debido principalmente a la escasa formación que reciben tanto durante el período universitario como posteriormente durante el ejercicio docente en los centros escolares (Colomo y Cívico, 2018; Pereira-Webber y Pereira-Webber, 2014; Puolimatka y Solasaari, 2006; Ransanz, 2014).

Es cierto que en los últimos tiempos se han dado algunas efemérides realmente significativas. Entre las mismas, destacar el logro a nivel universitario en el Departamento de Didáctica y Teoría de la Educación de la Facultad de Formación de Profesorado de la Universidad Autónoma de Madrid (el cual es el único donde se desarrolla la línea de investigación «Pedagogía de la muerte»), donde se impartió la materia «La muerte y su didáctica en Educación Infantil, Primaria y Secundaria», durante los cursos 2005-06 hasta el 2009-10, como asignatura de Libre Configuración en la formación de alumnos de Magisterio y Psicopedagogía. En esta misma línea, promovido por el Vicerrectorado de Participación Social de la Universidad Pablo de Olavide (Sevilla), durante el curso académico 20102011 se realizó un curso de libre configuración en torno a la temática de la educación para la finitud a través del cine. Además, fue parte integrante del programa de la asignatura optativa de Educación para la Salud dentro de la titulación de Educación Social desde el curso 2006-2007 en adelante (Pedrero, 2012). También es de destacar el Simposio 
de Historia de la Educación centrado en la Pedagogía de la Muerte, coordinado por Cagnolati y Hernández (2015), celebrado en la Universidad de Valladolid, donde diferentes expertos e investigadores aportaron estudios, comunicaciones y reflexiones sobre el papel que la educación debe y puede jugar respecto a la muerte.

Sin embargo, en la actualidad no existe una formación concreta y generalizada sobre pedagogía de la muerte para todos los egresados en las titulaciones de ciencias de la educación de las facultades españolas, no formando parte como tal del currículo universitario para su aprendizaje (salvo las excepciones anteriormente comentadas de la Universidad Autónoma de Madrid y de la Universidad Pablo de Olavide). Esta situación es radicalmente diferente en otros contextos internacionales, donde ya se han implementado y desarrollado programas para profesionales de la educación sobre la muerte (Harrawood, Doughty y Wilde, 2011), cuyos resultados apuntan a que dicho proceso formativo favorece la comprensión sobre la finitud y la mortalidad del ser humano, a la par que redujo la ansiedad ante la muerte. En esta misma línea tenemos a Schmidt (2007), quien tras obtener resultados favorables en estudios con profesionales que están en contacto con la muerte, afirmó que una mayor preparación y formación disminuye el grado de ansiedad ante la muerte. Por su parte, Herrán y Cortina (2006) evidenciaron una relación significativa entre el grado de reflexión y conocimiento de los profesores sobre la muerte y su actitud favorable a la normalización educativa de la misma.

Estas evidencias justifican la implementación de procesos formativos sobre el ámbito de la pedagogía de la muerte para profesionales de la educación, donde se incluya el desarrollo de destrezas, herramientas y habilidades que permitan reducir la ansiedad ante la condición finita del ser humano. En este sentido, una estrategia recurrente en disciplinas como la salud o las ciencias sociales es focalizar la atención en la dimensión emocional. La razón se sustenta en que las personas con capacidad para regular las emociones y al mismo tiempo comprender sus sentimientos y el de los demás, no tendrán problemas para expresar sus miedos o la dificultad será menor (Colell, 2005). Hay estudios que indican que el desarrollo de ciertas competencias motivacionales y emocionales (como la autoestima, el autoconcepto y la autoimagen) incrementan una de las habilidades que comporta la Inteligencia Emocional como es la competencia percibida, siendo necesaria para adaptarse adecuadamente a situaciones estresantes ya que promueve el sentimiento de autoeficacia, conllevando a que la persona evalúe las situaciones adversas como retos y se enfrente a ellas de manera activa (Fernández-Castro, 2007; Rueda y Pérez, 2004; Tang, Wu y Yan, 2002; Tomer y Elaison, 1996), reduciéndose considerablemente los niveles de ansiedad (Aradilla y Tomás-Sábado, 2006). Por esta razón, las futuras propuestas deberán estar conformadas por actividades que mejoren las diferentes competencias emocionales (autoconocimiento y comprensión de las emociones; aceptación de uno mismo; autocontrol; empatía; comunicación, etc.), con el fin de incrementar los niveles de éxito ante situaciones problemáticas, pudiendo así disminuir la ansiedad y estrés asociados a estos acontecimientos (Bellack, 1999; Caruso, Mayer y Salovey, 2002; Mayer, y Salovey, 1997) y a la vez favorecer el consiguiente desarrollo personal (Márquez-Cervantes y Gaeta-González, 2017).

Estas aportaciones junto con los resultados presentados en este estudio, confirman varias necesidades en las que profundizar: por un lado, desarrollar programas formativos desde el ámbito universitario con el fin de formar futuros docentes preparados para afrontar situaciones de duelo escolar; y por otro lado, incluir un plan de formación docente para los profesores en activo en los centros educativos, cuyo objetivo sea el fomento de las competencias emocionales para mejorar así la calidad del proceso de enseñanza-aprendizaje con sus estudiantes en torno al tema de la muerte, ya que es incuestionable la influencia que ejercen las habilidades emocionales en el proceso comunicativo, en la toma de decisiones, y en la capacidad de afrontamiento y resolución de los problemas con los discentes por parte de los profesionales de la educación.

Por último, el equipo de investigación es consciente de la limitación que implica utilizar un diseño transversal de tipo descriptivo, como es el caso, pero la ausencia de literatura científica en este campo de estudio y en este colectivo en concreto motivó el interés por explorar primeramente la naturaleza del estado emocional en el que se encuentra dicho grupo. La intención de este proyecto es que sirva como punto de partida para realizar posteriores investigaciones con una metodología correlacional o experimental. Se pretende aumentar el tamaño de la muestra para conseguir suficiente potencia estadística e incluir variables relacionadas con el estado emocional ante situaciones de muerte, como son la edad o el sexo, y que se encuentren equilibradamente representadas dentro de la muestra recogida (Limonero et al., 2010; Tomás-Sábado y 
Gómez-Benito, 2003), con el objetivo de realizar pruebas de significación estadística entre diferentes subgrupos y aportar datos con mayor validez científica. Ello permitirá poder profundizar en el grado de significación que presentan los factores de la escala y más concretamente los ítems de la misma, generalizar los resultados y proponer estrategias formativas ajustadas a la realidad de esta población sobre la pedagogía de la muerte, con el fin último de aportar mayor rigor científico en el campo de la educación.

\section{REFERENCIAS}

Abdel-Khalek, A. M. (2005). Death anxiety in clinical and nonclinical groups. Death Studies, 29(3), 251-259. doi: http://dx.doi.org/10.1080/07481180590916371

Abundis, V. (2016) Beneficios de las encuestas electrónicas como apoyo para la investigación. Tlatemoani. Revista Académica de Investigación, 22, 168-186.

Adamson, A. D. y Peacock, G.G. (2007). Crisis response in the public schools: A survey of school psychologists' experiences and perceptions. Psychology in the School, 44(8), 749-764. doi: http://dx.doi.org/10.1002/pits.20263

Alaminos, A. (2006). El muestreo en la investigación social. En A. Alaminos y J.L. Castejón. Elaboración, análisis e interpretación de encuestas, cuestionarios y escalas de opinión (pp. 46-67). Alcoy: Marfil.

Aradilla, A. y Tomás-Sábado, J. (2006). Efectos de un programa de educación emocional sobre la ansiedad ante la muerte en estudiantes de enfermería. Enfermería clínica, 16 (6), 321-326. doi: http://dx.doi.org/10.1016/S11308621(06)71239-6

Bellack, J. P. (1999). Emotional intelligence: a missing ingredient? Journal of Nursing Education, 38, 3-4. doi: https://doi.org/10.3928/0148-4834-19990101-03

Brown, J.A., Jimerson, S.R. y Comerchero, V.A. (2015). Cognitive Development Considerations to Support Bereaved Students: Practical Applications for School Psychologists. Contemp School Psychol, 19(3), 101-111. doi: https://doi.org/10.1007/s40688-014-0018-6

Cabadas, P. (2004). La muerte lúcida. Cómo encontrar sentido a la vida y a la muerte. Barcelona: Belacqva.

Cagnolati, A., y Hernández, J. L. (coords.). (2015). La Pedagogía ante la Muerte: reflexiones $e$ interpretaciones en perspectivas histórica y filosófica. Simposio de Historia de la Educación. Actas. Salamanca: Fahren House.

Caruso, D. R., Mayer, J. D. y Salovey, P. (2002). Relation of an ability measure of emotional intelligence to personality. Journal of Personality Assessment, 79(2), 306-320. doi: http://dx.doi.org/10.1207/S15327752JPA7902_12

Cavaye, J. y Watts, J. H. (2014). An integrated literature review of death education in pre-registration nursing curricula: key themes. International Journal of Palliative Care, 2014, 19. doi: http://dx.doi.org/10.1155/2014/564619

Colell, R. (2005). Análisis de las actitudes ante la muerte y el enfermo al final de la vida en estudiantes de enfermería de Andalucía y Cataluña. Tesis doctoral. Barcelona: Universitat Autònoma de Barcelona.

Colomo, E. (2016). Pedagogía de la Muerte y proceso de duelo. Cuentos como recurso didáctico. REICE. Revista Iberoamericana sobre Calidad, Eficacia y Cambio en
Educación, 14(2), 63-77. doi: http://dx.doi.org/10.15366/reice2016.14.2.004

Colomo, E. y Cívico, A. (2018). La necesidad de formación del profesorado en Pedagogía de la Muerte. Revista Electrónica Interuniversitaria de Formación del Profesorado, 21(1), 83-94. doi: http://dx.doi.org/10.6018/reifop.21.1.279961

Colomo, E. y Oña, J.M. (2014). Pedagogía de la Muerte. Las canciones como recurso didáctico. REICE. Revista Iberoamericana sobre Calidad, Eficacia y Cambio en Educación, 12(3), 109-121. Disponible en: http://www.redalyc.org/articulo.oa?id=55131318007

Cortina, M. y Herrán, A. (2011). Pedagogía de la Muerte a través del cine. Madrid: Universitas.

Feijoo, P. y Pardo, A. (2003). La escuela: una amiga en el duelo. Aula de Innovación Educativa, 122, 41-45.

Fernández-Castro, J. (2007). Alegría en la adversidad. El análisis de los factores que mantienen las emociones positivas en situaciones aversivas. En E. García Fernández-Abascal (Ed.), Emociones positivas (pp.217228). Madrid: Pirámide.

Gaona, J.M. (2015). El límite: una profunda investigación sobre la consciencia, el cerebro y las experiencias cercanas a la muerte. Madrid: La esfera de los libros.

Harrawood, L. K., Doughty, E. A. y Wilde, B. (2011). Death Education and Attitudes of Counselors-in-Training toward Death: An Exploratory Study. Counseling and Values, $56 \quad$ (1-2), 83-95. doi: https://doi.org/10.1002/j.2161-007X.2011.tb01033.x

Herrán, A. y Cortina, M. (2006). La muerte y su didáctica. Manual para educación infantil, primaria y secundaria. Madrid: Editorial Universitas.

Herrán, A. y Cortina, M. (2009). La muerte y su enseñanza. Diálogo Filosófico, 75, 499-516. Disponible en: https://laicismo.org/data/docs/archivo_1355.pdf

Kübler-Ross, E. (2006). Sobre la muerte y los moribundos. México, D.F: Random House Mondadori.

Letho, R. H. y Farchaus, K. (2009). Death anxiety: an analysis of an evolving concept. Research and theory for nursing practice, 23(1), 23-41.

Limonero, J. T. (1997). Ansiedad ante la muerte. Ansiedad y Estrés, 3(1), 37-46. Disponible en: https://www.researchgate.net/profile/Joaquin_Limonero 2/publication/235325305_Ansiedad_ante_la_muerte_De ath_anxiety/links/0912f510c37f22396b000000.pdf

Limonero, J. T., T.-Sábado, J., y Fernández Castro, J. (2006). Relación entre inteligencia emocional percibida y ansiedad ante la muerte de estudiantes universitarios. Ansiedad y Estres, 12(2-3), 267-278.

Limonero, J. T., Tomás-Sábado, J., Fernández-Castro, J., Cladellas, R. y Gómez-Benito, J. (2010). Competencia personal percibida y ansiedad ante la muerte en estudiantes de enfermería. Ansiedad y Estrés, 16(2-3), $177-188$.

López, R. (2016). Concepciones y actitudes ante la muerte: teorías implícitas, determinantes socioculturales y aspectos psicológicos relacionados. Tesis doctoral. Huelva: Universidad de Huelva.

Márquez-Cervantes, M. C. y Gaeta-González, M. L. (2017). Desarrollo de competencias emocionales en preadolescentes: el papel de padres y docentes. Revista Electrónica Interuniversitaria de Formación del Profesorado, 20(2), 221-235. doi: http://dx.doi.org/10.6018/reifop.20.1.232941

Mayer, J. D. y Salovey, P. (1997). What is emotional intelligence? En P. Salovey y D. Sluyter (eds.). Emotional development and emotional intelligence: 
Implications for educators (pp. 3-31). New York: Basic Books.

Moreno, Ma. V. (2013). Sentido de la vida y afectividad negativa (ansiedad, depresión y obsesión ante la muerte) en universitarios. Tesis doctoral. Badajoz: Universidad de Extremadura.

Osho (2004). Madurez. La responsabilidad de ser uno mismo. Barcelona: Grijalbo.

Pedrero, E. (2012). Educación para la salud y pedagogía de la muerte. Tesis doctoral. Sevilla: Universidad de Sevilla.

Pedrero, E. y Muñoz, M. (2008). La educación para la muerte como un reto emergente en la educación del siglo XXI: más allá de la educación para la salud. En F. López (Coord.). Educación como respuesta a la diversidad: una perspectiva comparada. Sevilla: SEEC (Universidad Pablo de Olavide).

Pereira-Webber, C. y Pereira-Webber, E. (2014). Confirming Life. Schools: Studies in Education, 11(1), 102-121. doi: https://doi.org/10.1086/675751

Peters, L., Cant, R., Payne, S., O'Connor, M., McDermott, F., Hood, K. y Shimoinaba, K. (2013). How death anxiety impacts nurses' caring for patients at the end of life: a review of literature. The open nursing journal, 7, 14. doi: https://doi.org/10.2174/1874434601307010014

Poch, C. (2009). La muerte nunca falla: un doloroso descubrimiento. Barcelona: Editorial UOC.

Poch, C. y Herrero, O. (2003). La muerte y el duelo en el contexto educativo. Barcelona: Paidós.

Puolimatka, T. y Solasaari, U. (2006). Education for Death. Educational Philosophy and Theory, 38(2), 201-213. doi: https://doi.org/10.1111/j.1469-5812.2006.00188.x

Ramos, A. (2016). El psicopedagogo como guía en los procesos de muerte y duelo en infantil. Ámbitos de Psicopedagía y Orientación, 45. Disponible en: http://ambitsdepsicopedagogiaiorientacio.cat/elpsicopedagog-com-a-guia-en-els-processos-de-mort-idol-infantil/

Ramos, A., Gairín, J. y Camats, R. (2018). Principios prácticos y funcionales en situaciones de muerte y duelo para profesionales de la educación. REICE. Revista Iberoamericana sobre Calidad, Eficacia y Cambio en Educación, 16(1), 21-33. doi: http://dx.doi.org/10.15366/reice2018.16.1.002

Ransanz, E. (2014). La muerte en la construcción de la identidad personal. Intervenciones educativas en la ESO. Tesis doctoral. Madrid: Universidad Complutense de Madrid.

Rodríguez, P., Herrán, A. y Cortina, M. (2012). Antecedentes de Pedagogía de la Muerte en España. Enseñanza \& Teaching. Revista Interuniversitaria de Didáctica, 30(2), 175-195.

Rodríguez, P., Herrán, A. y Cortina, M. (2015). Pedagogía de la muerte mediante aprendizaje servicio. Educación XXI, $18(1)$, 189-212.

doi: http://dx.doi.org/10.5944/educXX1.18.1.12317

Rueda, B. y Pérez García, A. M. (2004). Análisis comparativo de la competencia percibida general y la específica de salud. Ansiedad y Estrés, 10(1), 127-139.

Sabariego, M. (2012). El proceso de investigación. En Bisquerra, R. (coord.). Metodología de la investigación educativa (pp. 127 - 163). Madrid: La Muralla.

Schmidt, J. (2007). Validación de la versión española de la "Escala de Afrontamiento de la Muerte" y del "Perfil revisado de Actitudes hacia la Muerte": Estudio comparativo y Transcultural. Puesta en marcha de un programa de intervención. Tesis doctoral. Granada: Universidad de Granada.
Tang, C. S-K., Wu, A. M. S. y Yan, E. C. W. (2002). Psychological correlates of death anxiety among Chinese college students. Death Studies, 26(6), 491-499. doi: https://doi.org/10.1080/074811802760139012

Tomás-Sábado, J. y Gómez-Benito, J. (2003). Variables relacionadas con la ansiedad ante la muerte. Revista de Psicología General y Aplicada, 56(3), 257-279.

Tomás-Sábado, J. y Gómez-Benito, J. (2005). Construction and Validation of the Death Anxiety Inventory (DAI). European Journal of Psychological Assessment, 21(2), 108-114. doi: https://doi.org/10.1027/10155759.21.2.108

Tomás-Sábado, J. y Limonero, J. T. (2004). Ansiedad ante la muerte en estudiantes de enfermería: una comparación entre Egipto y España. Enfermería Clínica, 14, 328-333. doi: https://doi.org/10.1016/S1130-8621(04)73911-X

Wass, H. (2004). A Perspective on the Current State of Death Education. Death Studies, 28(4), 289-308. doi: https://doi.org/10.1080/07481180490432315

\section{Notas}

${ }^{1}$ Los ítems y factores del Inventario de Ansiedad ante la Muerte (Death Anxiety Inventory [DAI])

\begin{tabular}{lc}
\hline Ítems & Factores \\
\hline 1. Me angustia entrar en un cementerio. & 1 \\
2. La certeza de la muerte quita significado a la & 5
\end{tabular}
vida.

3. Me molesta oír hablar de temas relacionados 1 con la muerte.

4. Siempre me ha preocupado la posibilidad de 3 morir joven.

5. Me cuesta aceptar la idea de que todo acabe 4 con la muerte

6. Pienso que sería más feliz si ignorase que he 5 de morir.

7. Creo que tengo más miedo a la muerte que la 2 mayoría de las personas.

8. Me preocupa envejecer.

9. Me cuesta mucho aceptar que he de morir.

10. Nunca aceptaría trabajar en una empresa 1 funeraria.

11. Me asusta la idea de la nada después de la 4 muerte.

12. La idea de la muerte me provoca inquietud. 2

13. Muchas veces me pregunto cuál será la causa 3 de mi muerte.

14. Me gustaría vivir hasta una edad muy 2 avanzada.

15. Los ataúdes me ponen nervioso.

16. Me preocupa lo que haya después de la muerte.

17. A menudo pienso que puedo tener una 3 enfermedad grave.

18. La muerte es lo peor que puede sucederme. 2

19. Me impresiona mucho la visión de un 1 cadáver.

20. Con frecuencia pienso en mi propia muerte. 